\title{
Photometry of the Nearby Irregular Galaxy, NGC 6822*†
}

\author{
SUSAN E. KAYSER \\ Mt. Wilson and Palomar Observatories, Carnegie Institution of Washington, California Institute of Technology \\ and Lawrence Radiation Laboratory, Livermore, California
}

(Received 18 July 1966)

\begin{abstract}
NGC 6822 is a dwarf irregular in the Local Group at $\alpha=19^{\mathrm{h}} 42^{\mathrm{m}} \cdot 1, \delta=-14^{\circ} 53 ! 1$ (1950). The colormagnitude diagram is plotted from measures on photographic plates calibrated by a photoelectric sequence. Photometry is complete to $V=19^{\mathrm{m}} 5\left(M_{\mathrm{v}}=-5\right)$. Major features are bright blue supergiants, the brightest star having $M_{\mathrm{v}}=-9$, and a sequence of very red supergiants only one-half magnitude fainter than the blue, many of which have light variations lasting over 1000 days. A significant number of stars lie in the Hertzsprung gap. Two-color photometry shows there is intervening reddening of 0.27 . A luminosity function is calculated. Thirty-two variable stars are discussed, of which 13 are Cepheids, two are red semiregulars, one may be eclipsing, and the others are irregular. The 11 variables with $1^{\mathrm{m}} \cdot 6<(B-V)_{0}<2^{\mathrm{m}} 1$ have cycles of 200-2500 days. The period-luminosity relations for the Cepheids are $\langle V\rangle=23.35-2.97 \log P$ and $\langle B\rangle$ $=23.80-2.61 \log P$. These lead to a true distance modulus $(m-M)=23^{\mathrm{m}} \cdot 75 \pm 0^{\mathrm{m}}$. 15 . Secular changes in period are less than $0.001 \%$ in $40 \mathrm{yr}$.
\end{abstract}

\section{INTRODUCTION}

$\mathbf{N}$ GC 6822 is an irregular galaxy, of the Magellanic type. The coordinates are $\alpha=19^{\mathrm{h}} 42^{\mathrm{m}} \cdot 1$, $\delta=-14^{\circ} 53{ }^{\prime} .1$ (1950); the galactic coordinates are $l^{\text {II }}$ $=25^{\circ} 38, b^{\mathrm{II}}=-18.38$. Its long extension, the "bar," is aligned north-south, and subtends $20^{\prime}$, or $3.6 f \mathrm{kpc}$. ( $f$ is a correction factor, equal to the true distance of the nebula divided by the derived distance.) Radio observations of the $21-\mathrm{cm}$ line by Volders and Högbom (1961) show that the radial velocity with respect to the sun is $-50 \mathrm{~km} / \mathrm{sec}$; reduced to the local standard of rest it is $-23 \mathrm{~km} / \mathrm{sec}$. The galaxy is rotating about an axis with position angle $30^{\circ}$, to which no optical feature corresponds. Rotation curves indicate a mass of neutral hydrogen, $\mathfrak{M}_{\mathrm{H}}=1.9 \mathrm{f}^{2} \times 10^{8}$ solar masses, and a total mass, $\mathfrak{T}=1.7 f \times 10^{9}$ solar masses for a distance of $560 \mathrm{f} \mathrm{kpc}$ (assuming an inclination of $45^{\circ}$ to the plane of the sky).

The early research into NGC 6822 has been described by Hubble (1925) in a classic paper. He used Cepheid variables as distance indicators - the first application of the period-luminosity relation to regions outside the Galactic system. He also considered the diffuse nebulae, the luminosity function, and the total magnitude, as well as making comparisons with the Magellanic Clouds. Since this time, photoelectric photometry has provided us with an accurate system of magnitudes to much fainter limits than Hubble could go. (A comparison of Hubble's magnitudes with those in the present work indicate that his $m_{\mathrm{pg}}$ scale was systematically too bright for stars fainter than 16th mag. His " $18^{\mathrm{m}}$ " is about 19 mag on the $B$ system, and his " $19^{\mathrm{m}}$ " corresponds to $21 \mathrm{mag}$ in B.) Many additional plates have been taken by Hubble and Baade and, more recently, Arp, Oster-

* This work is adapted from the thesis submitted to the California Institute of Technology, Department of Astronomy, in partial fulfillment of the requirements for the degree of Ph.D.

† Part of this work was performed under the auspices of the U. S. Atomic Energy Commission.

$\ddagger$ Present address: The Belfer Graduate School of Science, Yeshiva University, New York. brock, and Sandage, as this investigation provides important basic data in analyzing the contents of near extragalactic space.

With this new material, and the increased resolution of the 200-in. telescope, are found improved periods for variable stars, light curves in two colors, and the color-magnitude diagram. We determine the periodluminosity relation of the 13 Cepheid variables, and derive the stellar luminosity function. The distance to the galaxy, linear dimensions, and density are deduced. Lastly, comparisons are made for each property between NGC 6822 and the other members of the Local Group.

\section{MATERIAL AND REDUCTION PROCEDURE}

\section{A. Photoelectric Material}

Dr. Arp has obtained a photoelectric sequence in NGC 6822 on the (UBV) system. Twenty-two stars were measured, since a magnitude system is better defined in practice by many stars measured once each than by a few stars known to great accuracy. Reductions were carried out by a standard computer program (Arp 1959). These primary standards are indicated by small Roman numerals on Plate II; their magnitudes are presented in Table I.

\section{B. Photographic Material}

From the collection of Mount Wilson and Palomar Observatories 139 photographic plates were obtained. Another six plates were lent by Dr. Hodge. In summary, there are

8 plates with $103 \mathrm{a}-\mathrm{O}+\mathrm{GG} 13$

20 plates with $103 \mathrm{a}-\mathrm{O}+\mathrm{GG} 1$ or WG 2

92 plates with $103 \mathrm{a}-\mathrm{O}$ or other blue-sensitive plate (some with silvered mirror)

120 total for blue magnitudes 
24 plates with $103 a-D+$ GG 11 or GG 14

1 plate with $103 \mathrm{a}-\mathrm{O}+\mathrm{UG} 2$

They cover a span of $40 \mathrm{yr}$.

Variable stars were detected by blinking pairs of plates on a blink-comparator. Hubble discovered 15 variables, Baade marked 9 others, and an additional 8 were found in the present work. They are identified on Plate II.

\section{Reduction Procedure}

All uncrowded stars with $V \leq 19^{\mathrm{m}} \cdot 5$ within a rectangle $14.1 \times 8.5$ enclosing the main body of the nebula were marked on a negative print and photometered for the color-magnitude diagram. This rectangle was divided into five concentric sections with similar proportions, labeled A, B, $\cdots, \mathrm{E}$, with areas in the ratio $1: 1: 2: 2: 4$, A being the smallest, innermost section. In each section, stars in the northwest corner were numbered 1-49, stars in the southwest corner were labeled 51-99, stars in the northeast corner were 101-149, and stars in the southeast were 151-199. About 17 stars in D and 28 in $\mathrm{E}$ brighter than $19^{\mathrm{m}} .5$ were inadvertently omitted because their visual magnitudes were estimated too faint from the print. In two quarters there were more than 50 stars; numbers greater than 200 were applied to the excess. Thus every star has a letter and a number. Identification charts for the stars are Plates II-V of UCRL-14801-T, a Lawrence Radiation Laboratory Report (see the second paragraph of the acknowledgments). A dozen stars measured in the $\mathrm{H}$ II region in the northwest are labeled F, and are shown on Plate II.

To minimize effects due to unresolved background stars, the three best, lightly exposed plates were selected in $B$ and in $V$. (Only one plate on the $B$ system was lightly exposed; so for the other two, plates taken with the GG 1 filter had to be used.) All the stars with $V \leq 19^{\mathrm{m}} \cdot 5$, as well as the standards and the variables, were measured on these six plates with the Mt. Wilson and Palomar Observatories' Sartorius variable-iris astrophotometer. Photometer readings for the standards were plotted against photoelectric magnitudes and a calibration curve was drawn for each plate. For each standard, the magnitude corresponding to the photometer reading was read from the calibration curve. These magnitudes, termed "photographic" (as opposed to "photoelectric"), were averaged for the $B$ and $V$ plates. Since they define a calibration curve with much less scatter than the photoelectric values, they were used henceforth; this results in more consistent work. The theoretical color equation derived by Arp (1960b) was applied to the average of the blue magnitudes to transform it to the (UBV) system (see Sec. D for further discussion). Distance-to-center effects introduced by coma were negligible. The adopted photographic magnitudes are tabulated in Table I. (The identification of the star described by the photoelectric values for $\mathrm{xvi}$ is uncertain. However, the adopted
TABLE I. Magnitudes of the primary standards.

\begin{tabular}{|c|c|c|c|c|c|c|}
\hline \multirow[b]{2}{*}{ Star } & \multicolumn{3}{|c|}{ Photoelectric values } & \multirow[b]{2}{*}{$n^{\mathrm{a}}$} & \multicolumn{2}{|c|}{$\begin{array}{l}\text { Adopted } \\
\text { photographic } \\
\text { values }\end{array}$} \\
\hline & $V_{\mathrm{pe}}$ & $(B-V)_{\mathrm{pe}}$ & $(U-B)_{\mathrm{pe}}$ & & $V_{\mathrm{pg}}$ & $(B-V)_{\mathrm{pg}}$ \\
\hline i & $12^{\mathrm{m}} \cdot 26$ & $0 \mathrm{~m} 74$ & $0^{\mathrm{m}} \cdot 23$ & 12 & $12^{\mathrm{m}} \cdot 26$ & $0^{\mathrm{m}} \cdot 74$ \\
\hline ii & 16.13 & 1.09 & 0.57 & 1 & 16.12 & 1.06 \\
\hline iii & 17.50 & 1.51 & 1.02 & 1 & 17.32 & 1.70 \\
\hline iv & 19.07 & 1.06 & 0.05 & 1 & 19.06 & 1.08 \\
\hline $\mathrm{v}$ & 18.20 & 0.98 & & 1 & 18.27 & 1.07 \\
\hline vi & 20.39 & 0.71 & -0.10 & 1 & 20.19 & 0.77 \\
\hline vii & 15.91 & 1.04 & 0.71 & 4 & 15.99 & 0.96 \\
\hline viii & 18.22 & 0.85 & & 1 & 18.36 & 0.72 \\
\hline ix & 19.78 & 1.13 & & 2 & 20.11 & 0.80 \\
\hline$x$ & 17.28 & 0.88 & 0.18 & 1 & 17.31 & 0.88 \\
\hline$x i$ & 16.99 & 0.87 & 0.31 & 1 & 16.91 & 0.86 \\
\hline xii & 13.15 & 0.66 & 0.12 & 3 & 13.15 & 0.66 \\
\hline xiii & 15.82 & 0.92 & 0.54 & 1 & 15.86 & 0.91 \\
\hline xiv & 16.37 & 1.02 & 0.50 & 1 & 16.35 & 1.03 \\
\hline $\mathrm{xv}$ & 21.21 & 0.60 & & 1 & 21.15 & 0.71 \\
\hline xvi & 20.50 & 0.35 & & 1 & 20.64 & 1.22 \\
\hline xvii & 13.40 & 0.66 & 0.14 & 3 & 13.40 & 0.66 \\
\hline xviii & 17.30 & 1.11 & 0.56 & 2 & 17.28 & 1.08 \\
\hline xix & 19.48 & 0.79 & & 2 & 19.55 & 0.99 \\
\hline$x x$ & 19.87 & 1.79 & & 1 & 19.63 & 1.86 \\
\hline $\mathrm{xxi}$ & 20.82 & 0.74 & & 1 & 20.57 & 1.14 \\
\hline xxii & 17.27 & 0.71 & 0.11 & 1 & 17.23 & 0.75 \\
\hline
\end{tabular}

a $n$ is number of times star was observed.

magnitudes are photographic and there is no doubt as to which star was measured for xvi on the plates.)

New calibration curves were drawn with the adopted photographic values, and average magnitudes and colors for all the stars measured on the six plates were derived with the help of an IBM 7094. They are listed in Table 3 of UCRL-14801-T.

Since the stars fainter than $19.5 \mathrm{mag}$ can be seen only on heavily exposed plates, where background effects are very large, it is necessary to select a faint sample of stars from regions away from the center. We see on the photographs that NGC 6822 has an extension from the main bar to the southeast and another to the east. Stars fainter than $19^{\mathrm{m}} 5$ were selected in each of these regions and numbered consecutively. Those in the east are labeled G; those in the southeast (partly overlapping with section $\mathrm{E}$ ) are labeled $\mathrm{H}$. To reduce background difficulties still further, stars in these regions, whose magnitudes had been determined in the bright star work, were used as secondary standards. Finding charts are Plates VI and VII of UCRL-14801-T, respectively. Three heavily exposed plates in $B$ and in $V$ were measured and reduced as before. Average magnitudes and colors of stars in the faint sample are found in Table 3 of UCRL-14801-T.

Because most of the plates are exposed so long that background effects are significant, stars were chosen to serve as local standards around each variable and their magnitudes found from the six lightly exposed plates. These secondaries were measured with the variables and primary standards on all the remaining plates. Reductions were carried out (by hand) in the same manner described. 


\section{Discussion of Errors}

Examination of the plots of deviations of standards from the calibration curve $\Delta B$ against color index indicate an internal consistency of

$$
\begin{aligned}
& \pm 0^{\mathrm{m}} \cdot 15 \text { in } V \text { or } B \\
& \pm 0.20 \text { in } B-V \quad \text { (single measure) }
\end{aligned}
$$

and an uncertainty of

$$
\begin{aligned}
& \pm 0^{\mathrm{m}} 03 \text { in } V \text { or } B \\
& \pm 0^{\mathrm{m}} .04 \text { in } B-V \quad \text { (final average value) }
\end{aligned}
$$

for the 78 primary and secondary standards (based on averages of 24 visual and 19 blue plates). Systematic errors, however, may be more sizeable. We consider several possible sources of error:

(1) The zero point of the $B$ and $V$ scales. This depends upon an average of 12 photoelectric observations of the standard star $i$, made on eight nights. If the extinction coefficients used in the standard reductions to the $(U B V)$ system are correct for the large zenith angle of NGC 6822 (secant $z \sim 1.5$ ), the zero point is probably known to $\pm 0 \mathrm{~m} \cdot 04$. The photoelectric standards determine the scale down to $V=21$ and $B=21^{\mathrm{m}} .8$. Values fainter than this are obtained by extrapolating the linear part of the calibration curve for a heavily exposed plate.

(2) The color system for very blue stars. As there were no photoelectric standards with $(B-V)<0^{\mathrm{m}} \cdot 6$, the magnitudes of a few blue stars were determined from 103a-O+GG 13 plates to extend the color range. Plots of $\Delta B$ against color index showed that GG 13 and GG 1 magnitudes agreed systematically to $\pm 0^{\mathrm{m}} \cdot 05$ for $(B-V)>0$ m.0. However, for very blue stars, we expect a color divergence between the two, so the quantity $[0.044-0.088(B-V)]$ (Arp 1960b; Swope 1963) was added to the colors obtained by differencing the blue and the visual photographic magnitudes, to transform them to the $(U B V)$ system. [The $(B-V)$ in this expression is the uncorrected color]. This was done only for stars in A through $F$, since magnitudes in $G$ and $\mathrm{H}$ depend solely on GG 13 plates. Variables are not affected by the color equation because they are all in the well-determined range of color index. The very red stars are subject to analogous uncertainties, however, as the reddest photoelectric color is $1 \mathrm{~m} .8$. Similar arguments show that we expect the deviation of tabulated $(B-V)$ from the true color to be at most $0^{\mathrm{m}} \cdot 1$ by $(B-V)_{\text {tab }}=2^{\mathrm{m}} \cdot 6$.

(3) The calibration curves. Iris diameters are reproducible to the equivalent of 0.002 , even for plates with poor image quality, but the relation between the iris diameter and magnitude (i.e., the calibration curve) is uncertain by \pm 0 m. 05 . It is this which contributes a large part of the internal inconsistency. For stars near the plate limit, this uncertainty may be \pm 0.25 or

\begin{tabular}{|c|c|c|c|c|c|c|c|}
\hline Star & $(U-B)_{\mathrm{pg}}$ & $(B-V)_{\mathrm{pg}}$ & $E_{y}$ & $V_{\mathrm{pg}}$ & $M_{\mathrm{v}}$ & $r(\mathrm{pc})$ & Notes \\
\hline$i$ & $0 \frac{\mathrm{m}}{24}$ & $0 \mathrm{~m} 74$ & $0 \mathrm{~m} 06$ & $12^{\mathrm{m}} \cdot 26$ & $5 \mathrm{~m} \cdot 16$ & 246 & $\mathbf{a}$ \\
\hline $\mathrm{ii}$ & 0.61 & 1.06 & 0.29 & 16.12 & 5.65 & 832 & a \\
\hline iii & 1.02 & 1.70 & & & & & $a, b$ \\
\hline iv & 0.20 & 1.08 & 1.28 & & & & a, c \\
\hline $\mathrm{v}$ & 0.66 & 1.07 & 0.27 & 18.27 & 5.81 & 2180 & \\
\hline $\mathrm{vi}$ & 0.03 : & 0.77 & & & & & $a, c$ \\
\hline vii & 0.71 & 0.96 & 0.05 & 15.99 & 6.31 & 832 & a \\
\hline viii & 0.30 & 0.72 & 0.37 : & 18.36 & 3.01: & 7090: & $\mathrm{d}$ \\
\hline $\mathrm{ix}$ & $-0.16:$ & 0.80 & 1.06 & & & & c \\
\hline$x$ & 0.14 & 0.88 & 1.05 & & & & $a, c$ \\
\hline$x i$ & 0.32 & 0.86 & 0.19 & 16.91 & 5.03 & 1820 & $\mathrm{a}$ \\
\hline xii & 0.10 & 0.66 & 0.12 & 13.15 & 4.39 & 501 & a \\
\hline xiii & 0.56 & 0.91 & 0.08 & 15.86 & 5.97 & 852 & a \\
\hline xiv & 0.71 : & 1.03 & 0.16 : & 16.35 & 6.13 & 892 & a \\
\hline $\mathrm{xv}$ & $>-0.06$ & 0.71 & & & & & c \\
\hline$x v i$ & $>-0.64$ & 1.22 & & & & & e \\
\hline xvii & 0.14 & 0.66 & 0.02 & 13.40 & 4.89 & 468 & a \\
\hline xviii & 0.61 & 1.08 & 0.32 & 17.28 & 5.58 & 1413 & $\mathrm{a}$ \\
\hline xix & $-0.22:$ & 0.99 & & & & & c \\
\hline$x x$ & 0.17 : & 1.86 & & & & & e \\
\hline$x x i$ & $>-0.75:$ & 1.14 & & & & & e \\
\hline xxii & 0.32 : & 0.75 & 0.08 & 17.23 & 5.00 & 2460 & $a, f$ \\
\hline G $\quad 1$ & 0.23 & 0.00 & & & & & \\
\hline G 2 & 1.23 & 1.06 & & & & & \\
\hline G 3 & 0.62 & 0.72 & & & & & \\
\hline G 4 & $0.45:$ & 1.22 & & & & & \\
\hline G 5 & 1.04 & 1.08 & & & & & \\
\hline G 6 & $-0.24:$ & -0.31 & & & & & \\
\hline G 7 & 0.16 : & 0.51 & & & & & \\
\hline G 8 & $0.49:$ & 0.49 & & & & & \\
\hline A 68 & 0.20 : & 0.73 & 0.08 & 15.00 & 4.91 & 935 & \\
\hline B174 & -0.80 & 0.18 & $0.50:$ & 19.00 & $-4.24:$ & $2 \times 10^{5}:$ & $\mathbf{g}$ \\
\hline C 38 & 0.39 & 1.02 & & & & & c \\
\hline C 41 & 0.39 & 0.78 & 0.00 & 13.56 & 5.70 & 372 & \\
\hline C 47 & 0.95 & 1.29 & 0.41 & 16.11 & 6.17 & 550 & \\
\hline C 48 & $-0.83:$ & 0.98 & & & & & \\
\hline C 79 & 1.18 & 2.29 & & & & & b \\
\hline C126 & 0.18 & 0.88 & & & & & e \\
\hline C178 & 0.15 & 0.67 & 0.02 & 15.39 & 4.92 & 1202 & \\
\hline C196 & -0.02 & 0.50 & & & & & c \\
\hline D 33 & 0.25 & 0.83 & 0.26 & 15.89 & 4.45 & 1350 & \\
\hline D 55 & 0.44 & 0.94 & 0.22 & 15.37 & 5.35 & 741 & \\
\hline D122 & 0.25 & 0.88 & & & & & c \\
\hline D123 & 0.27 & 0.99 & & & & & c \\
\hline D124 & 0.69 & 1.10 & 0.30 & 16.30 & 5.85 & 814 & \\
\hline D154 & 0.16 & 0.78 & & & & & c \\
\hline D156 & 0.06 & 0.25 & 0.29 & 15.60 & 0.34 & 5785 & $\mathrm{~h}$ \\
\hline E 19 & 1.13 & 1.68: & & & & & b \\
\hline E 22 & 0.14 & 0.81 & & & & & c \\
\hline E 31 & 0.19 & 0.84 & & & & & c \\
\hline E101 & 0.98 & 1.13 & 0.13 & 17.30 & 6.70 & 1098 & \\
\hline E102 & 0.32 & 0.80 & 0.08 & 17.14 & 5.35 & 2041 & \\
\hline E103 & 0.41 & 0.96 & 0.28 & 15.81 & 5.12 & 934 & \\
\hline E106 & 1.07 & 1.17 & 0.11 & 16.15 & 6.90 & 602 & \\
\hline E107 & 0.23 & 0.73 & 0.04 & 16.05 & 5.16 & 1412 & \\
\hline E118 & 0.03 & $0.72:$ & & & & & c \\
\hline E122 & 1.23 & 1.17 & & & & & e \\
\hline E123 & 0.10 & 0.69 & & & & & c \\
\hline E142 & 0.72 & 1.12 & 0.30 & 16.20 & 5.89 & 759 & \\
\hline E161 & 0.35 & 0.47 & 0.29 & 16.10 & 1.99 & 4436 & $\mathrm{~h}$ \\
\hline
\end{tabular}
more.
(4) Coma, crowding, and background light. Each of these three causes the luminosity to be overestimated. However, the color-magnitude stars were measured

TABLE II. Reddening from the two-color plot.

a $(U-B)_{\text {pe }}$ available

b Probably an $\mathrm{M}$ star. The unreddened sequence is not well determined here

S Stars that fall in this region, above the $(B-V)_{0}=0.56$ trajectory, cannot be reliably unreddened.

Dif

Did not fall on any

s $(U-B)$ pe was used.

$\mathbf{b}$ The most likely of the two possibilities was chosen. 
from plates on which these effects are very small, and the use of local standards corrects for them on the other plates. The uncertainty of reduction due to these conditions is about \pm 0.07 and is the source of the rest of the internal inconsistency.

In sum, values for the color-magnitude diagram (means of three plates in each color) are accurate to

$\pm 0^{\mathrm{m}} \cdot 10$ in $V$ or $B(V<21.0, B<21.8)$

\pm 0.14 in $B-V \quad(B-V>0.0) \quad$ "estimated error"

\pm 0.20 in $B-V \quad(B-V<-0.3)$.

Stars brighter than $16 \mathrm{mag}$ are overexposed on the plates, so their magnitudes cannot be better determined than this. Stars fainter than 19m5 were measured on heavily exposed plates so they were well above the plate limit and their magnitudes are as well determined as the majority, which lie between 16 and $19^{\mathrm{m}} \cdot 5$.

\section{REDDENING}

The color excess $E_{y}$ was determined by translating stars in the $(U-B, B-V)$ plot back along the reddening trajectories (Wildey 1963). To supplement the photoelectric data, 30 bright stars selected from the outer regions of the nebula were measured with all the primary standards on the one $U$ photographic plate. As the $U_{\mathrm{pg}}$ values agreed very well with the $U_{\mathrm{pe}}$ for the 14 primaries, the photographic magnitudes were used throughout for consistency. $(U-B)_{\mathrm{pg}}$ values may be found in Table II.

The two-color plot of these stars, Fig. 1, shows that most of the points fall in a band to the right of the unreddened luminosity class $\mathrm{V}$ main sequence (Johnson 1958; Johnson and Iriarte 1958; Arp 1958) with $(B-V)_{0}>0$ m. The assumption is made that on the average these stars should be traced back to the red portion of the class $\mathrm{V}$ curve. The secondary standards from $\mathrm{G}$ lie to the left of the unreddened curve. If their $B$ values were made fainter by 0.14 , these stars would lie on or to the right of the unreddened main sequence. Such correction would also reduce the number of excessively blue stars in the faint sample from $G$, but since the magnitudes for these were derived in the same way as for all other stars, there seems to be no reason for a systematic difference to exist.

After the color excesses had been determined, the distance to each star, given in Table II, was calculated from

$$
5 \log r_{\mathrm{pc}}=V-M_{\mathrm{v}}-3.0 E_{\mathrm{y}}+5,
$$

where the mean $M_{\mathrm{v}}-(B-V)_{0}$ relation is taken from Allen (1963, p. 199). We may wonder if the extremely large color excesses apparently found for stars lying above the $(B-V)_{0}=0^{\mathrm{m}} .56$ trajectory (see Fig. 1) are meaningful. Considering that the $(U-B)$ and $(B-V)$ values are uncertain to \pm 0 . 14 , the "true observed colors" of these stars could easily lie below this critical reddening line. If the slope of the trajectories used was

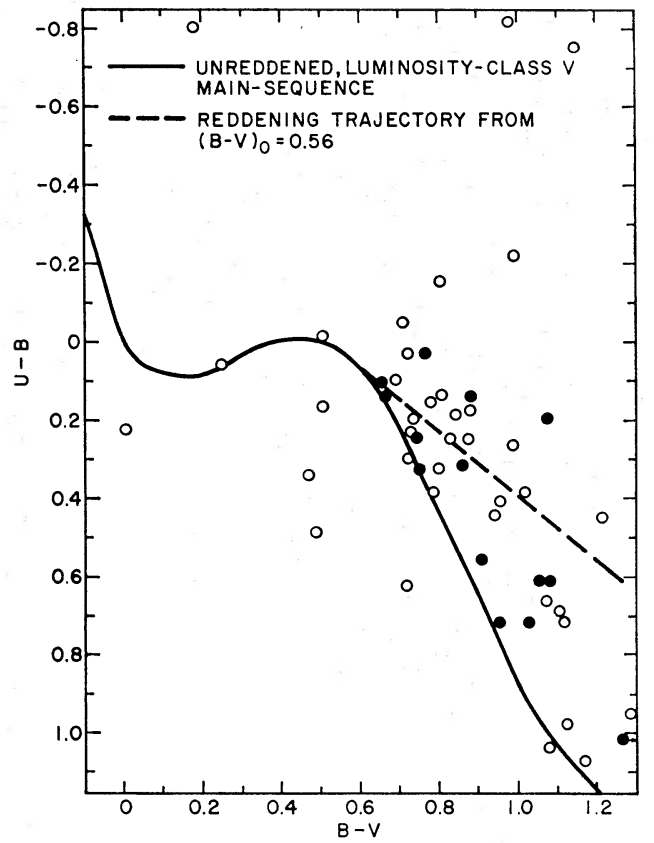

FIG. 1. The two-color plot of field stars. Dots are stars with $U_{\text {pe }}$; open circles are stars with $U_{\mathrm{pg}}$ only.

made shallower, many of these stars could be traced back to the red portion of the unreddened line. Furthermore, an ultraviolet excess in some stars would lead to their unreddened positions lying above the "knee" of the normal unreddened curve. We conclude that the true color of any star bluer than $0^{\mathrm{m}} \cdot 6$ is very uncertain.

When the reddening is plotted against distance (Fig. 2) for the stars in Table II, we see that $E_{y}$ increases with distance, reaching a final value of

$$
E_{y}=0.27 \pm 0.03 \text {. }
$$

This occurs at a distance of 800 to $1200 \mathrm{pc}$ along the line of sight, corresponding to a distance from the Galactic plane of 240 to $360 \mathrm{pc}$. This is somewhat greater than the mean half-width of the absorbing layer, 150 pc (Allen 1963, p. 269).

Assuming the absorption $A_{v}=3.0 E_{y}$,

$$
A_{v}=0.8 \pm 0.1
$$

with a mean rate of absorption of $0.7 \mathrm{mag} / \mathrm{kpc}$. (Usual

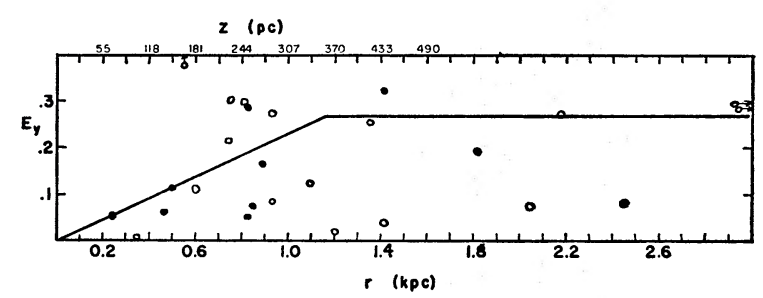

Fig. 2. Reddening as a function of distance. Dots are stars with $U_{\mathrm{pe}}$; open circles are stars with $U_{\mathrm{pg}}$ only. $\left(U_{\mathrm{pg}}\right.$ was used for all stars.) The lower scale shows distance along the line of sight; the upper scale is distance from the Galactic plane. 


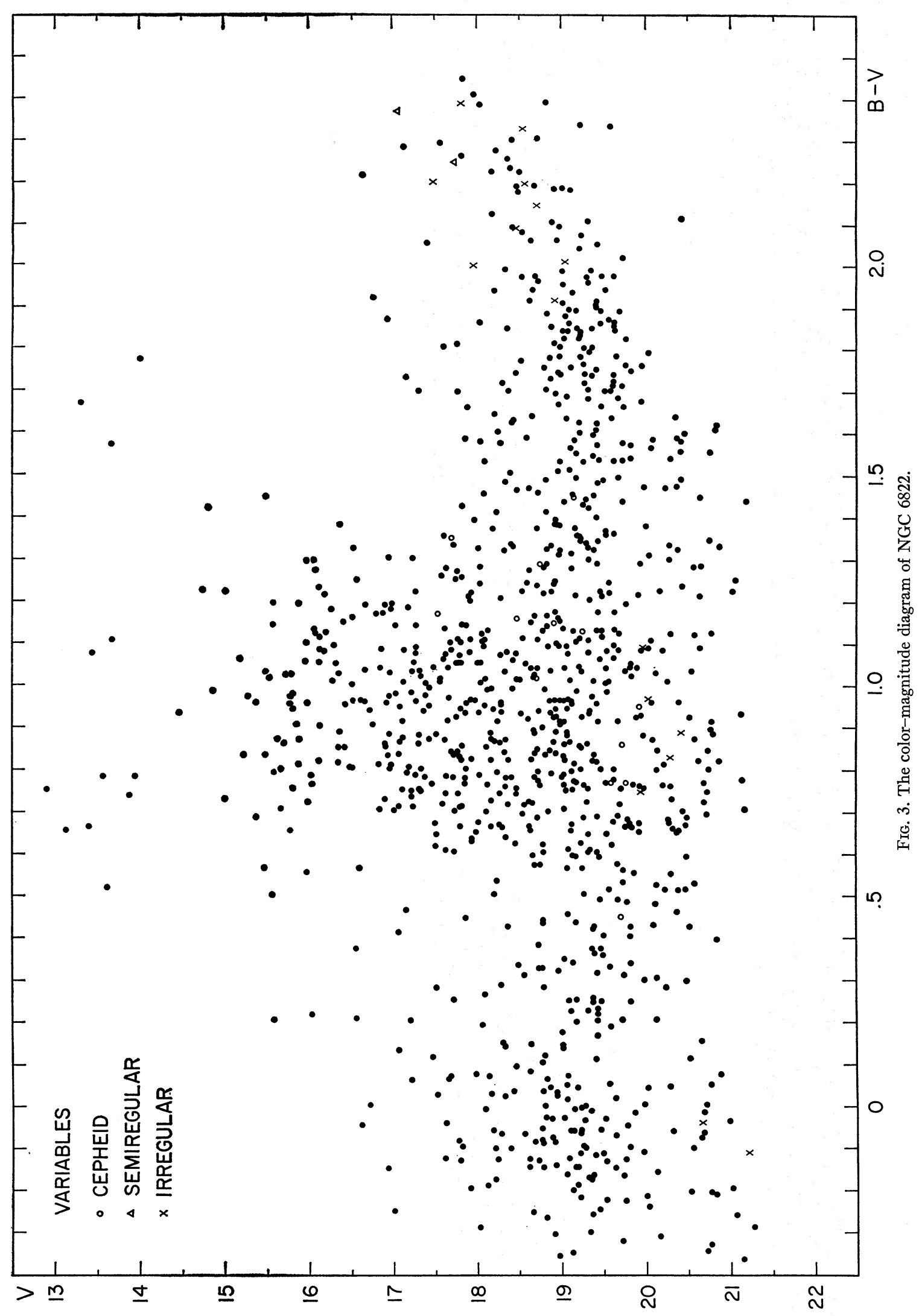


mean rates range from 0.6 to $1.0 \mathrm{mag} / \mathrm{kpc}$.) We may compare this with the cosecant reddening (Arp 1962)

$$
\begin{aligned}
& E_{\mathbf{y}}=0^{\mathrm{m}} \cdot 058 \sec b=0^{\mathrm{m}} \cdot 18, \\
& A_{\boldsymbol{v}}=0^{\mathrm{m}} \cdot 6 .
\end{aligned}
$$

Agreement to $30 \%$ indicates that the assumptions that the stars came from luminosity class $\mathrm{V}$ with $(B-V)_{0}>0 \times 56$ were justified.

No differential reddening was found across the nebula but the data do not exclude a change of as much as $0^{\mathrm{m}} \cdot 10$ in $E_{y}$ from one side to the other.

\section{COLOR-MAGNITUDE DIAGRAM}

Stars lie in three groups in the color-magnitude diagram, Fig. 3. (All stars of Tables I, and Table 3 of UCRL-14801-T are plotted.) The blue sequence looks very like the top of the $h / \chi$ Persei main sequence (Wildey 1964): a broad fan curving back toward the red for the brightest stars. Blue stars are found with equal frequency in regions $\mathrm{A}, \mathrm{B}$, and $\mathrm{C}$. There are fewer in $\mathrm{D}$, and none in $\mathrm{E}$ except in the southeast quarter. The bluest stars are in $\mathrm{C}$ and $\mathrm{F}$; there are also some very blue stars in the eastern extension.

The red stars form a sequence beginning at $(B-V)_{\text {obs }}$ $=1^{\mathrm{m}} \cdot 5$ and go out to $2^{\mathrm{m}} \cdot 5$, getting brighter as they get redder. A large number of variable stars are found at the red tip of the sequence. There are equal frequencies of red stars in regions $\mathrm{A}, \mathrm{B}$, and $\mathrm{C}$, perhaps increasing from $\mathrm{A}$ to $\mathrm{C}$ and favoring the west side. $\mathrm{C}$ contains the reddest stars as well as the bluest (and the brightest of each color). Again, the percentage decreases in D and there are none in $\mathbf{E}$ except in the southern extension. Indeed, the lack of red and blue stars in $\mathrm{E}$ is very striking.

The central hump, $0^{\mathrm{m}} \cdot 5<B-V<1^{\mathrm{m}} \cdot 5$, lies in the middle of the Hertzsprung gap, where we would expect very few stars other than the Cepheid variables which fall across it. Arguments for and against its being composed of foreground stars are presented in the next section. It may be noted that the hump starts quite clearly at $(B-V)_{\mathrm{obs}}=0 \cdot 6$, except in the $\mathrm{E}$ region (again excluding the southern extension) where it starts at 0.8 . The density of stars in the hump decreases redward of $(B-V)_{\mathrm{obs}}=1{ }^{\mathrm{m}} \cdot 0$.

\section{FIELD STARS}

Determination of the number of foreground stars, necessary for calculating the luminosity function, has been approached from three angles.

\section{A. Star Counts in Selected Areas}

Star counts made by Seares et al. (1925) and collated by van Rhijn (1929) are presented in Table III for Selected Areas 135 and 136, for the smoothed average at $\left|b^{\mathrm{I}}\right|=20^{\circ}$, and for the smoothed average interpolated
TABLE III. Field star counts.

\begin{tabular}{rrrrrrrr}
\hline \multicolumn{9}{c}{$A_{m}{ }^{\mathrm{a}}$} \\
\multicolumn{1}{c}{ S.A. 135} & $-16^{\circ}, 351^{\circ}$ & S.A. $136\left|b^{\mathrm{I}}\right|=20^{\circ}$ & Total & Hump \\
\hline 13 & 4 & 4 & 2 & 2 & 2 & 2 \\
14 & 10 & 10 & 5 & 5 & 5 & 5 \\
15 & 32 & 26 & 14 & 10 & 7 & 7 \\
16 & 81 & 61 & 21 & 20 & 20 & 20 \\
17 & 187 & 118 & 48 & 36 & 60 & 50 \\
18 & 627 & 235 & 100 & 63 & 119 & 87 \\
19 & $\cdots$ & 462 & $\cdots$ & 105 & 214 & 129 \\
\hline \hline
\end{tabular}

a Stars per magnitude interval in 0.033 square degrees.

to $\left(-16^{\circ}, 351^{\circ}\right)$, the coordinates of NGC 6822 in van Rhijn's system. Seares' magnitude scale has been transformed to the $B$ system by

$$
\begin{aligned}
& B=0.944 m_{\mathrm{pg}}+0.177, \quad B<16, \\
& B=1.777 m_{\mathrm{pg}}-2.696, \quad B>16,
\end{aligned}
$$

a relation deduced from Johnson $(1951,1953)$, correcting for the scale divergence found for S.A. 68 by Stebbins, Whitford, and Johnson (1950). The counts are reduced to the area of the rectangle enclosing the main bar (0.033 square degrees). For comparison, the counts from NGC 6822 are included, in column 6. Although the number of field stars is uncertain by a factor of 2, it is evident that all stars in the rectangle with $B<16$ mag must be foreground stars.

\section{B. A Constructed Field Star Color-Magnitude Diagram}

In order to discriminate by color, we construct a field star color-magnitude diagram. The $m-\log \pi$ method was used (Bok 1931) in which the integral over distance,

$$
A_{m}=\omega \int_{0}^{\infty} \phi\left(M_{\mathrm{v}}\right) \rho(r) r^{2} d r
$$

TABle IV. Assumed variation of absorption and density with distance.

\begin{tabular}{rrrrrrrr}
\hline \hline$k$ & $r(\mathrm{pc})$ & $z(\mathrm{pc})$ & $R(\mathrm{kpc})^{\mathrm{a}}$ & $A_{v}$ & $E_{y}$ & $2+\log \rho$ & $\log \rho V$ \\
\hline 10 & 100 & 24 & 9.92 & $0^{\mathrm{m}} 0$ & $0 \mathrm{~m} 0$ & 1.98 & 0.81 \\
11 & 159 & 42 & 9.87 & 0.1 & 0.0 & 1.96 & 1.27 \\
12 & 252 & 71 & 9.78 & 0.2 & 0.1 & 1.94 & 1.84 \\
13 & 398 & 117 & 9.66 & 0.3 & 0.1 & 1.89 & 2.39 \\
14 & 631 & 191 & 9.46 & 0.4 & 0.1 & 1.77 & 2.88 \\
15 & 1000 & 307 & 9.15 & 0.7 & 0.2 & 1.57 & 3.27 \\
16 & 1585 & 491 & 8.67 & 0.8 & 0.3 & 1.23 & 3.54 \\
17 & 2515 & 783 & 7.91 & 0.8 & 0.3 & 0.81 & 3.72 \\
18 & 3980 & 1245 & 6.80 & 0.8 & 0.3 & 0.49 & 4.00 \\
19 & 6310 & 1980 & 5.28 & 0.8 & 0.3 & 0.03 & 4.13 \\
20 & 10000 & 3140 & 4.30 & 0.8 & 0.3 & -0.4 & 4.3 \\
21 & 15850 & 4980 & 7.40 & 0.8 & 0.3 & -0.8 & 4.5 \\
22 & 25152 & 7900 & 15.45 & 0.8 & 0.3 & -1.2 & 4.7 \\
23 & 39800 & 12520 & 29.0 & 0.8 & 0.3 & -1.6 & 4.9 \\
24 & 63130 & 19880 & 51.0 & 0.8 & 0.3 & -2.0 & 5.1 \\
25 & 100000 & 31530 & 86.0 & 0.8 & 0.3 & -2.4 & 5.3 \\
\hline \hline
\end{tabular}

a $R=$ distance from center of Galaxy, assuming the sun is $10 \mathrm{kpc}$ from center. 
TABLE V. Field star color-magnitude distribution.

\begin{tabular}{rrrrrrrr}
\hline \hline$B-V$ & 19 & 18 & 17 & 16 & 15 & 14 & 13 \\
\hline 0.1 & 2 & 1 & 1 & & 1 & & \\
0.2 & 3 & 2 & & & 1 & & \\
0.3 & 6 & 2 & 2 & 2 & 1 & 2 & 1 \\
0.4 & 8 & 5 & 3 & 2 & 2 & & 2 \\
0.5 & 7 & 4 & 3 & 2 & 1 & 2 & \\
0.6 & 4 & 3 & 3 & 1 & 1 & 2 & 1 \\
0.7 & 12 & 10 & 7 & 3 & 3 & 2 & 1 \\
0.8 & 24 & 18 & 12 & 10 & 3 & 1 & \\
0.9 & 23 & 15 & 11 & 4 & 1 & 1 & \\
1.0 & 14 & 8 & 7 & 4 & 1 & & \\
1.1 & 15 & 8 & 4 & 2 & 1 & & 1 \\
1.2 & 16 & 11 & 5 & 4 & 3 & & \\
1.3 & 14 & 7 & 3 & 1 & 1 & & \\
1.4 & 10 & 8 & 2 & 2 & 1 & 1 & \\
1.5 & 9 & 6 & 3 & 3 & & & \\
1.6 & 11 & 4 & 2 & & 1 & & \\
1.7 & 9 & 2 & & & 1 & & \\
1.8 & 1 & 1 & & & & & \\
1.9 & 1 & 1 & 1 & 1 & & & \\
2.0 & & & & 1 & & & \\
2.1 & & & & & & \\
2.2 & 3 & 1 & 1 & & 1 & & \\
\hline
\end{tabular}

Number of stars between $V-\frac{1}{2}$ and $V+\frac{1}{2}$; and $B-V$ and $B-V+0.1$.

is replaced by a sum over shells:

$$
A_{m}=\sum_{k=0}^{\infty} \phi\left(m_{\mathrm{v}}-k+5-A_{k}\right) \rho(k) V(k) .
$$

Here $A_{m}$ is the number of stars in solid angle $\omega$ with apparent magnitude $m_{\mathrm{v}}-\frac{1}{2}<m_{\mathrm{v}}<m_{\mathrm{v}}+\frac{1}{2}$ (cut off at $\left.m_{\mathrm{v}}=19 \mathrm{mag}\right), k=5 \log r, A_{k}$ is the total absorption in front of the $k$ th shell, $\rho(k)$ is the density of stars $/ \mathrm{pc}^{3}$ (Allen 1963, p. 240) normalized to unity at the Galactic plane, and $V(k)$ is the volume of the $k$ th shell. Table IV shows the assumed variation of absorption, reddening and density with distance. For $\phi\left(M_{\mathrm{v}}\right)$ - the density of stars $/ \mathrm{pc}^{3}$ with $M_{\mathrm{v}}-\frac{1}{2}<M_{\mathrm{v}}<M_{\mathrm{v}}+\frac{1}{2}$-the solar neighborhood luminosity function (Allen 1963, p. 238) was used in all shells. As a result, about 15 stars too few were calculated for $V=19 \mathrm{mag}$, but this is a small error compared with that introduced by the necessary

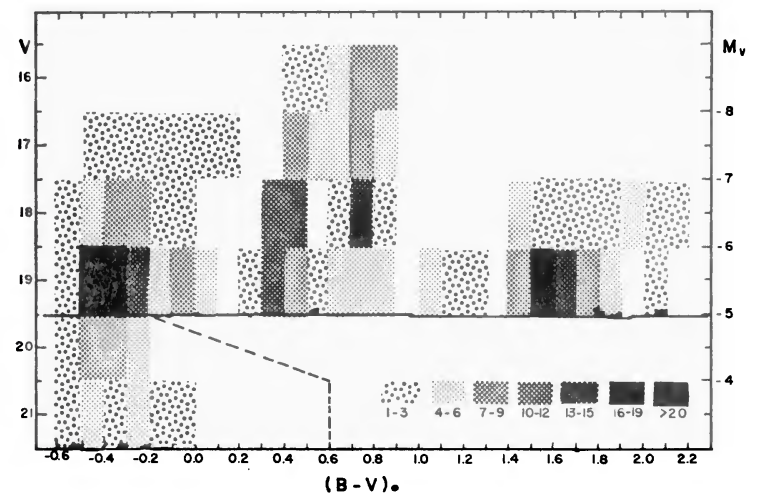

FIG. 4. Number of stars in the color-magnitude diagram in intervals of $\left[\Delta V=1^{\mathrm{m}}, \Delta(B-V)=0 \mathrm{~m} 1\right]$, after subtracting the entire constructed field star diagram. extrapolation of the density out to $100 \mathrm{kpc}$. Since the density is known only for $r<4 \mathrm{kpc}$, considerable uncertainty is entailed in its extrapolation. Stars were distributed in color according to Starikova (1960). (This distribution is the most probable one, but not the only possibility.) The results are presented in Table V. The number of stars calculated in each magnitude interval by this method is given in Table VI, column 2 . Comparison with the actual counts in the galaxy (column 3) shows that the field star diagram is overpopulated by $50 \%$, probably due to overestimating the density at large distances. When the field star distribution (slightly smoothed to remove statistically invalid fluctuations) is subtracted from the observed color-magnitude diagram, the intrinsic distribution remains. This is presented in Fig. 4.

Table $\mathrm{V}$ establishes that most of the field stars lie between $0^{\mathrm{m}} \cdot 4<B-V<1^{\mathrm{m}} \cdot 6$; that is, they are in the central hump. They are too few, however, to account for all the stars actually observed in this range of color index, particularly when the overpopulation of the field star diagram is allowed for, as we see in Table VI.

\section{Comparison of Inner and Outer Sections}

Since the outer section $\mathrm{E}$ contains a much smaller percentage of NGC 6822 stars than the inner section A, a comparison of the color-magnitude diagrams of the outer and inner regions, normalized to equal areas, furnishes the possibility of measuring directly the density of field stars in our own galaxy in the direction of NGC 6822. By subtracting the counts of E (omitting the southeast quarter of $\mathrm{E}$ to get a purer sample of field stars) from those in A for each magnitude or color interval, we subtract out the field stars from A. (There are too few stars to permit a statistically valid difference of their actual color-magnitude diagrams to be made.) Insofar as $\mathrm{E}$ does contain intrinsic stars, these are perforce subtracted too, so we have a lower limit to the true distribution in $\mathrm{A}$. The counts (A-E) by magnitude intervals are listed in Table VII, column 6; and the counts by color are presented in Fig. 5. The histogram shows that a significant number of stars are present between $0^{\mathrm{m}} \cdot 2<(B-V)_{0}<0^{\mathrm{m}} \cdot 6$, in the Hertzsprung gap,

TABLE VI. Counts from NGC 6822 and from constructed field star diagram.

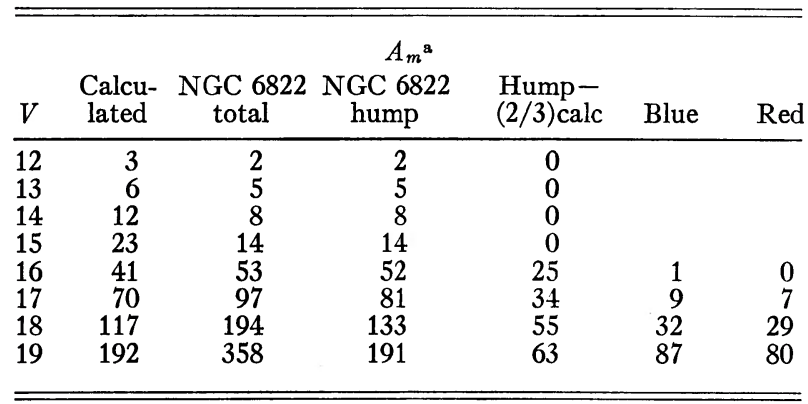

* Stars per magnitude interval in 0.033 square degrees. 
TABLE VII. Luminosity function.

\begin{tabular}{|c|c|c|c|c|c|c|c|c|}
\hline \multirow[b]{2}{*}{$V$} & \multirow[b]{2}{*}{$M_{\mathrm{v}}$} & \multicolumn{3}{|c|}{ All sections $\mathrm{A}$ to $\mathrm{E}$} & \multicolumn{3}{|c|}{ Section A } & \multirow{2}{*}{$\begin{array}{c}\text { Galactic } \\
\text { plane } \\
10+\log \phi(M)^{\mathrm{b}}\end{array}$} \\
\hline & & No. of stars ${ }^{\mathrm{a}}$ & Stars $/ \mathrm{pc}^{3}$ & $10+\log \phi(M)$ & No. of stars $^{\mathrm{a}}$ & Stars $/ \mathrm{pc}^{3}$ & $10+\log \phi(M)$ & \\
\hline 16 & -8.5 & 26 & $87 \times 10^{-10}$ & 1.94 & & & & \\
\hline 17 & -7.5 & 50 & 167 & 2.24 & $<1$ & $<90 \times 10^{-10}$ & $<2.0$ & 1.64 \\
\hline 18 & -6.5 & 116 & 389 & 2.59 & 15 & 1410 & 3.15 & 2.15 \\
\hline 19 & -5.5 & 226 & 760 & 2.88 & 51 & 4800 & 3.68 & 2.63 \\
\hline
\end{tabular}

clustering against the blue edge of the Cepheid instability region. From this comparison of (A-E) with A, we conclude that of the stars with $0.5<(B-V)_{\text {obs }}$ $<0 \mathrm{~m} 9,55 \% \pm 5 \%$ are intrinsic; of the hump stars redder than this, fewer than $4 \%$ are intrinsic.

\section{STELLAR CONTENT}

\section{A. Color-Magnitude Diagram}

When the field stars are removed, the color-magnitude diagram loses the scatter of stars brighter than $16 \mathrm{mag}$, and the hump becomes much less dense (see Fig. 4). The blue stars must be at the top of the main sequence; there is a very strong resemblance to the fan of supergiants in the color-magnitude diagram of $h / \chi$ Persei (Wildey 1964) as shown in Fig. 10. The similarity does not extend to the red supergiants, for NGC 6822 has almost as many red stars as blue (90 red to 110 blue), and the red stars are only a half-magnitude fainter than the blue, while $\mathrm{h} / \chi$ Persei itself has no red supergiants and the few found in its association are not as bright as in the nebula core. Moreover, the $\mathrm{h} / \chi \mathrm{M}$ supergiants get fainter with increasing color index, whereas the NGC 6822 red stars brighten. The two systems differ also in the Hertzsprung gap: $\mathrm{h} / \chi$ Persei has no stars there.

The M31 color-magnitude array for Field IV (Swope 1963) shows great similarity with the present one. However, Field IV has fewer very bright stars, particularly among the extremely red ones and among the blue ones (which are fainter in M31 than the red stars). We can see in Field IV how the red and blue sequences approach each other at fainter magnitudes.

The color-magnitude diagram for stars in the field of the Large Magellanic Cloud (Wooley 1963; Bok et al. 1967) also has common features with that of NGC 6822, but it is hard to distinguish these features because of greater contamination by field stars. The blue sequences are alike, but the LMC red stars are relatively scarce, although they become as red as those in this work. Wooley's findings from proper motion studies, that virtually all the stars in the LMC between $0^{\mathrm{m}} \cdot 5<(B-V)_{\text {obs }}<1^{\mathrm{m}} \cdot 5$ are foreground stars, are not repeated in NGC 6822, as Sec. VC shows.

H II regions are prominent in the nebula. Sérsic's (1959) angular measures of the three largest (identified on Plate II) correspond to diameters of

$$
\text { NGC } 6822141 \text { (III) } 116 \text { (X) } 114 \text { (I) pc. }
$$

These are smaller than the ones in the Clouds:

$\begin{array}{llll}\text { LMC } & 290 & 185 & 115 \\ \text { SMC } & 185 & 135 & 135 \mathrm{pc} .\end{array}$

In summary, the stellar content of NGC 6822 is much like that of other young Population I systems, with a large number of blue supergiants of magnitude $-5 \mathrm{mag}<M_{\mathrm{v}}<-8 \mathrm{mag}$, having a spread in color $-0^{\mathrm{m}} .5<(B-V)_{0}<+0^{\mathrm{m}}$.1. It differs in having a much larger proportion of red stars of comparable brightness. Since red and blue stars are always found together in similar numbers throughout the nebula, it is likely they are different evolutionary stages of one type of star. Therefore, the red stars are also Population I. As for stars of intermediate color, they are probably as frequent as blue stars, and tend to lie in the colormagnitude diagram near the blue edge of the Cepheid region.

\section{B. Luminosity Function}

Only a small portion of the luminosity function can be obtained. For the galaxy as a whole, after subtracting two-thirds (to allow for the overpopulation discussed earlier) of the constructed field star diagram counts from the observed counts, we find that the number of stars is halved in each brighter magnitude interval (see Table VI, column 3). For the inmost section A (subtracting counts from E), the decrease is more rapid, implying that the center is not the part of the galaxy richest in very bright stars. We may interpret this to mean that present star formation is most common not

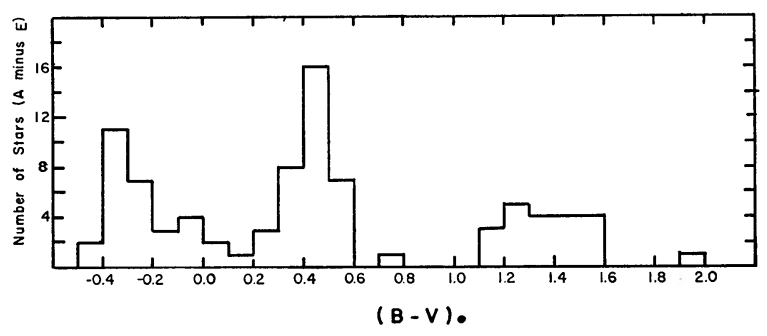

FIG. 5. Counts of stars in section A as a function of $(B-V)_{0}$, after subtracting counts from $\mathrm{E}$ (omitting the southeast quarter of $\mathrm{E}$ and normalizing to equal areas). 

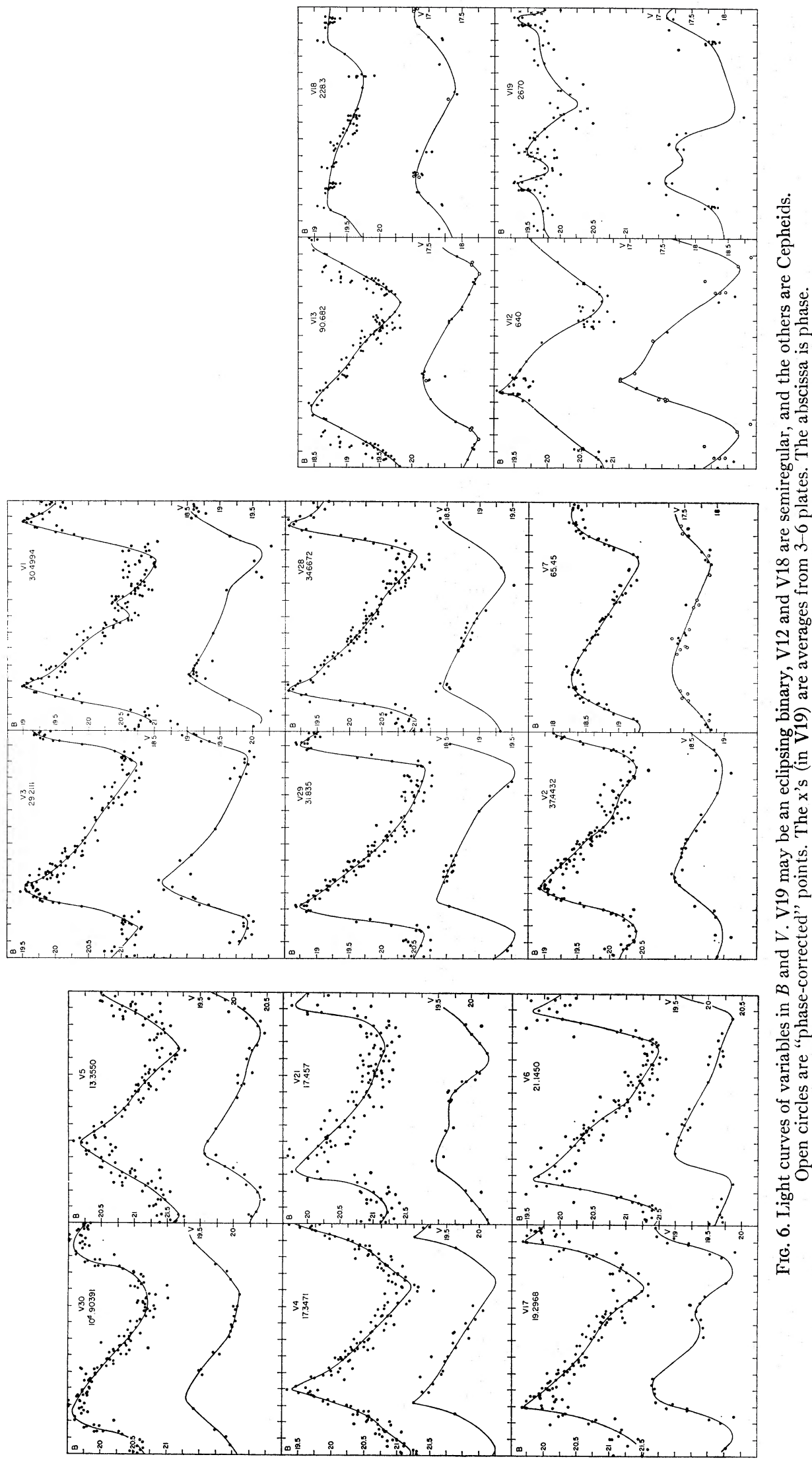
at the center but in section $\mathrm{C}$, where the fraction of very red and very blue stars is greatest.

We can get an approximation to the volume of the galaxy by assuming its dimension in the line of sight is equal to the mean apparent diameter. If we assume an inclination to the plane of the sky of $45^{\circ}$ and a distance of $560 \mathrm{f} \mathrm{kpc}$, then the volume filled by stars in the large rectangle is

$$
V=3 f^{3} \times 10^{9} \mathrm{pc}^{3} .
$$

This is probably good to a factor of 2 . The luminosity function $\phi(M)$ is now calculated in Table VII. When we compare this to the luminosity function valid for the plane of our galaxy (Starikova 1960) we see that NGC 6822 has proportionally more very blue stars. [The slope of $\phi(M)$ in NGC 6822 is 2-2.5; in our galaxy it is 3. In the LMC it is also 3 (Shapley 1943, p. 79).] Moreover, blue stars are as a whole twice as dense there as in our own spiral arms. (The relative density of all stars per $\mathrm{pc}^{3}$ is unknown.) The central density is about five times the nebular average.

\section{Integrated Magnitude}

The integrated magnitude of all stars measured here brighter than $19 \mathrm{~m} \cdot 5$ in the main body of the nebula is $9.56 \pm 0.05(V)$ or $10.48 \pm 0.05(B)$. Stars fainter than this contribute another 0 m.06. This leads to an integrated color of 0.92 . However, the numerous foreground stars will dominate this figure because they include the brightest stars in the line of sight. The integrated color of the central section A is 0.75 , and the color of the faint sample is 0.71 . This is probably close to the true value since field stars will be relatively scarce in these regions. Averaging these two numbers and subtracting the foreground reddening gives us the adopted unreddened color

$$
(B-V)_{0}=0 \cdot 46 \pm 0^{\mathrm{m}} \cdot 07 .
$$

This is comparable to that of the Magellanic Clouds: 0.50 (LMC) and 0 m.35 (SMC) (de Vaucouleurs 1964).

For the total magnitude we may use Holmberg's (1957) value of integrated $m_{\mathrm{pg}}=9^{\mathrm{m}} \cdot 21$. We correct $m_{\mathrm{pg}}$ to the $B$ system by $B=0.944 m_{\mathrm{pg}}+0.177$. With the adopted color, this is equivalent to $V=8^{\mathrm{m}} \cdot 14$, or $L_{v}=2.8 f^{2} \times 10^{8} L \odot$.

From Volders and Högbom (1961), the mass is $1.7 f \times 10^{9} \mathrm{~N} \odot$ (probably satisfactory to a factor of 2 ) so the mass-to-light ratio is

$$
\mathfrak{T} / L_{v} \approx 6.1 f^{-1} .
$$

This value is typical of Population I: in the solar neighborhood the ratio is 1.7 , and in the Clouds it is 2.0 (Limber 1960). By contrast, in the Sb galaxy M31, the ratio is 16, and in E-type galaxies it is at least 200 .

\section{VARIABLE STARS}

Twenty-nine stars detected by blinking plates, and seven others whose magnitudes from the color-magni- tude plates showed poor agreement, were studied for variation. Thirteen are Cepheids, two are red semiregulars, one may be an eclipsing binary, and no periods could be found for the others. Four of Hubble's "Cepheids" (V8, V9, V10, and V11), are in this last group. Five stars are probably not variable.

The list is expected to be complete for all variable stars in the large rectangle with $V \leq 19^{\mathrm{m}} 5$ and amplitude greater than 0.5 . Smaller amplitudes cannot be unambiguously distinguished from accidental error due to background effects. The degree of completeness is unknown for stars outside these limits. Counts of variable stars (Kukarkin and Parenago 1958; Plaut and Soudan 1962) indicates that, at most, two variables may be expected from the foreground.

\section{A. Variable Stars}

V1. Cepheid. The $V$ curve is poorly delineated except at maximum.

V2. Cepheid. The period seems to be somewhat smaller than average for the first 9000 days by 0 d 0002 .

V3. Cepheid.

V4. Cepheid.

V5. Cepheid. The rise to maximum is slow.

V6. Cepheid. The point of maximum is not well determined. The period for the first few years of observation is $0.3 \%$ greater than average.

V7. Cepheid. This long-period star exhibits phase changes of \pm 0.15 ; that is, when the magnitudes are plotted against phase $=(\mathrm{JD}-2420000) / P$, three or four parallel curves appear, separated by $\approx 0.15$ in phase. Some phase changes are positive, others negative. For clarity, only the first five years are used in plotting the $B$ light curve in Fig. 6 . All visual magnitudes have been corrected to the "initial phase" in plotting the $V$ light curve.

V8. Irregular. Hubble called this a Cepheid, but it is much too blue. Variations are short term, very small, and do not confirm Hubble's period.

V9. Irregular. Hubble called this a Cepheid, and it has the right color and shape of light curve, but no period has been found that satisfies the data. Maxima can occur as little as three, or as much as ten, days apart.

V10. Irregular. This is another of Hubble's "Cepheids" for which his period was not verified. Although this star lies among the Cepheids in the color-magnitude diagram, it appears to have a small 5-day variation superimposed on a 630-day cycle with larger amplitude.

V11. Irregular. Hubble called this a Cepheid, but it is too blue. There is a small amplitude variation of 3.5 day. This star is very faint, and the data are not reliable.

V12. Semiregular. This extremely red star has two modes of behavior. Most of the time it varies regularly with a period of 640 days, but every three to six cyles, it does something else for about 180 days. No plates 
TABLE VIII. Nonregular variables.

\begin{tabular}{lccrlll}
\hline \hline Star & $\bar{V}$ & $\bar{B}$ & $(B-V)_{0}$ & \multicolumn{1}{c}{$\Delta B$} & \multicolumn{1}{c}{$\Delta V$} & \multicolumn{1}{c}{ Type of variation } \\
\hline V14 & $17 \mathrm{~m} 82$ & $20^{\mathrm{m} 21}$ & $2^{\mathrm{m} 12}$ & $1^{\mathrm{m} 3}$ & $1 \mathrm{~m} 1$ & Long \\
V18 & 17.07 & 19.44 & 2.10 & 0.54 & 0.62 & Semiregular; $P=228.3$ \\
V32 & 18.53 & 20.86 & 2.06 & 1.3 & 0.9 & Medium \\
V12 & 17.74 & 19.99 & 1.98 & 1.57 & 1.81 & Semiregular; $P \sim 640$ \\
V19 & 17.49 & 19.69 & 1.93 & 0.95 & 1.05 & Eclipsing binary (?); \\
& & & & & & $P=2670$ \\
V23 & 18.56 & 20.76 & 1.93 & 1.2 & 1.6 & Medium \\
V31 & 18.47 & 20.56 & 1.82 & 1.1 & 0.5 & Medium \\
V22 & 19.04 & 21.05 & 1.74 & 1.5 & 2.3 & Medium-long \\
V15 & 17.97 & 19.97 & 1.73 & 0.9 & 0.7 & Long \\
V26 & 18.92 & 20.84 & 1.65 & 1.1 & 0.8 & Medium \\
V27 & 19.97 & 21.06 & 0.82 & 1.5 & 0.8 & Medium \\
V24 & 20.03 & 21.00 & 0.70 & 0.8 & 0.9 & Short \\
V25 & 20.41 & 21.30 & 0.62 & 1.4 & 0.8 & $P=13.33(?)$ \\
V10 & 20.28 & 21.11 & 0.56 & 1.8 & 1.8 & Medium-long \\
V9 & 19.93 & 20.68 & 0.48 & 1.4 & 0.7 & Short \\
V 8 & 20.67 & 20.63 & -0.31 & 0.9 & 0.7 & Short \\
V11 & 21.22 & 21.11 & -0.38 & 0.8 & 0.9 & Short \\
& & & & & & \\
\hline \hline
\end{tabular}

* Type of variation: Long $\sim 2500$ days, medium $\sim 30$ days, short $\sim 3$ days.

were taken during such hiatuses, so this mode cannot be more clearly described. The blue light curve in Fig. 6 is drawn for the first three cycles; the $V$ light curve is constructed by phase correcting, as with V7.

V13. Cepheid. This is the longest period Cepheid found in the nebula, with the maximum coming later in $V$ than in $B$. Like $\mathrm{V} 7$, it shows phase changes, although not as markedly, which tend to occur every eighth cycle. Only points with the "initial phase" have been plotted for the $B$ light curve in Fig. 6 . When necessary, $V$ points have been phase corrected.

V14. Irregular. This very red star has a slow variation of 2400 days. It might be semiregular.

V15. Irregular. There are small amplitude variations, lasting at least 250 days. It is very red.

V16. Irregular. This star is below the plate limit most of the time. It reaches maximum every eight days, on the average.

V17. Cepheid. This star is much too red for its period.

V18. Semiregular. There are phase changes of \pm 0.10 in this red star. Only the first five years have been plotted in the $B$ light curve, Fig. 6. Points on the $V$ curve have been phase corrected.

V19. Eclipsing(?). Real periodicity seems to be present here, although the light curves of this bright, red star have a large scatter. For clarity, averages of three-six days are plotted as mean points in Fig. 6.

V20. Irregular. Like V16 this is a very faint star. Maxima are spaced about 10 days apart.

V21. Cepheid. There is more scatter than usual about the $B$ light curve.

V22. Irregular. Variations are at least 26 days, and less than 2600 days. The amplitude is large, especially in $V$.

V23. Irregular. This red star has variations lasting about two weeks.

V24. Irregular. It has erratic variations of 10 days or less.
V25. Irregular(?). A period of 13.33 days may fit the data, but there is a very large scatter, perhaps because the star is near the faint limit. If this is a true period, the light curve shows a secondary maximum.

V26. Irregular. Cycles last less than 25 days.

V27. Irregular. Like V9, this star has the right color and shape of light curve to be a Cepheid but it is not periodic. Cycles average 14 days.

V28. Cepheid.

V29. Cepheid.

V30. Cepheid. This is extremely bright and blue for its period. (There may be a connection between its color and the presence of many other blue stars in the eastern extension.) The $V$ curve has no points at maximum. This has the shortest period found so far in the nebula.

V31. Irregular. Variations of this red star last 8 to 30 days.

V32. Irregular. The $B$ amplitude of this red star is large. Cycles average 30 days.

A131, A159, and A6 were studied for variation, but the amplitudes are small enough to be explained by background effects. The last of these may be variable, with a five-day cycle. The secondary standard E282 also may be variable. A star in the eastern extension, G55, has very bad agreement among the three magnitudes measured for the color-magnitude diagram; this is a possible variable.

The amplitudes, mean magnitudes, and unreddened colors of the irregular variables, the semiregulars, and the eclipsing binary are collected in Table VIII. Most of them lie along the red sequence and share in its characteristic of getting brighter with increasing redness; they tend to have cycles lasting about 500-2500 days. The light curves of NGC 6822 red irregulars are not known well enough to permit close comparison with local red variables such as $\alpha$ Ori $\left(M_{\mathrm{v}}=-5^{\mathrm{m}} \cdot 9, P \sim 2070\right.$ days), $\mu$ Cep ( $M_{\mathrm{v}}=-3 \mathrm{mag}, P \approx 750$ days) or S Per (double period of 810 and 916 days) (Campbell 1939), but they give an impression of being considerably more erratic. Other irregulars lie among the Cepheids in the color-magnitude diagram, with cycles of 10-30 days. The two very blue ones have three-day variations.

\section{B. Cepheids}

Light curves were drawn in $B$ and in $V$; they are seen in Fig. 6. Despite the "phase changes," secular changes in period were less than $0.001 \%$ in $40 \mathrm{yr}$ $(<0.036 \%$ for V7,$<0.010 \%$ for V13). The difference between the mean curves was plotted as the $B-V$ curve, and the mean unreddened color, $\left\langle(B-V)_{0}\right\rangle_{\mathrm{mag}}$, was found. The $B$ curve was transformed into an intensity curve to calculate the mean $\langle B\rangle$, following Kraft's (1961a) rules. The mean $\langle V\rangle$ was derived from $\langle V\rangle$ $=\langle B\rangle-\langle B-V\rangle_{\mathrm{mag}}+C(\Delta B)$, where $C(\Delta B)$ was taken from Kraft. The properties of the Cepheids are presented in Table IX. (Mean magnitudes can be determined to \pm 0 m.03, mean colors to $\pm 0^{\mathrm{m}} \cdot 05$.) 
PHOTOMETRY OF NGC 6822

TABLE IX. Properties of the Cepheid variables.

\begin{tabular}{|c|c|c|c|c|c|c|c|c|}
\hline Star & $P$ & $\log P$ & $\langle V\rangle$ & $\langle B\rangle$ & $\left\langle(B-V)_{0}\right\rangle_{\mathrm{mag}}$ & $\Delta V$ & $\Delta B$ & $(m-M)^{\mathrm{a}}$ \\
\hline V13 & $90 \mathrm{~d} 682$ & 1.957 & $17^{\mathrm{m}} 75$ & $19^{m} \cdot 05$ & $1 \mathrm{~m} 08$ & $0 \mathrm{~m} 84$ & 1935 & 0.35 \\
\hline V 7 & 65.45 & 1.816 & 17.56 & 18.70 & 0.90 & 0.58 & 1.04 & 0.29 \\
\hline V 2 & 37.4432 & 1.573 & 18.52 & 19.62 & 0.89 & 0.76 & 1.48 & 0.27 \\
\hline V28 & 34.6672 & 1.540 & 18.85 & 20.03 & 1.02 & 0.96 & 1.96 & 0.19 \\
\hline V29 & 31.835 & 1.503 & 18.80 & 19.72 & 0.75 & 1.20 & 1.90 & 0.17 \\
\hline V 1 & 30.4994 & 1.484 & 19.02 & 20.05 & 0.88 & 1.08 & 2.03 & 0.23 \\
\hline V 3 & 29.2111 & 1.466 & 19.32 & 20.38 & 0.86 & 1.30 & 1.70 & 0.25 \\
\hline V 6 & 21.1450 & 1.325 & 19.87 & 20.54 & 0.50 & 0.87 & 1.91 & 0.24 \\
\hline V17 & 19.2968 & 1.285 & 19.22 & 20.58 & 1.18 & 1.22 & 1.80 & 0.28 \\
\hline V21 & 17.457 & 1.242 & 19.76 & 20.57 & 0.59 & 0.81 & 1.37 & 0.25 \\
\hline V 4 & 17.3471 & 1.239 & 19.67 & 20.35 & 0.50 & 1.25 & 1.82 & 0.38 \\
\hline V 5 & 13.3550 & 1.126 & 19.98 & 20.87 & 0.68 & 0.86 & 1.48 & 0.43 \\
\hline V30 & 10.9039 & 1.038 & 19.73 & 20.15 & 0.18 & 0.82 & 1.14 & 0.32 \\
\hline
\end{tabular}

a $(m-M)$ is the fraction of the period from minimum to maximum ( $B$ curve).

The period-amplitude diagram, plotted in Fig. 7, resembles that of our own system more than that of the SMC (Arp 1960a). A comparison with $V$ amplitudes of Cloud Cepheids (Gascoigne and Kron 1965), however, shows greater similarity. Interpretation of the periodcolor relation is more difficult. We may plot $\left\langle(B-V)_{0}\right\rangle_{\mathrm{mag}}$ against $\log P$ for 31 Cepheids in our Galaxy (Kraft 1961b), for 63 Cepheids in the SMC (Arp 1960a) (these 94 stars are represented by the dashed line in Fig. 7), for 27 Cepheids in both Clouds (Gascoigne and Kron 1965) (represented by crosses), and for the 13 Cepheids in NGC 6822 (represented by filled circles). Fernie's (1963) correction to Kraft's colors is included, making them about 0 m.04 redder. Arp (private communication) believes there is 0 m.06 greater reddening than he previously assumed for the SMC, and this has been allowed for. Examination of these relations shows that the mean period-color lines ("bands" is more accurate, since their width is $0^{\mathrm{m}} \cdot 2$ ) for Galactic Cepheids and Arp's SMC Cepheids agree to within $\pm 0^{\mathrm{m}} \cdot 05$ in $\langle B-V\rangle$. The stars with $\log P>1.5$ in NGC 6822 and the Clouds fall along a continuation of this line. The clump of stars at $\log P \approx 1.2$, which are 0.2 bluer than the mean relation, is populated by stars from all three irregular galaxies and by $\mathrm{Y}$ Oph in our own. These stars have no special significance in the period-amplitude or period-luminosity diagrams. The three stars with $\log P<1.0$ in the Gascoigne and Kron paper lie about $0 \mathrm{~m} .2$ above the mean line and may represent a sequence distinct from the mean line. Even without separate sequences, the considerable width of the period-color relation indicates that the characteristics of Cepheids are not determined by one parameter alone (e.g., period), which weakens the reliability of Cepheids as distance indicators. For clarity, Fig. 7 contains the mean line only for the Galactic and SMC variables. The stars V30 and V17 (which do not have large amplitude defects) are very blue and red, respectively. V30 falls above the mean period-luminosity line, as expected of a blue star, but V17 does not fall below the line. It appears from Fig. 7 and from the shape of the light curves that Cepheids in NGC 6822 are like those in our Galaxy.

The period-luminosity diagram is drawn in Fig. 8. A least-squares solution was made, omitting V30 in $B$ and half-weighting it in $V$. Slopes of $-2.98 \pm 0.29(V)$ and $-2.53 \pm 0.29(B)$ were obtained, which almost coincide with those of Gascoigne and Kron (1965) for the Clouds. These are not necessarily in disagreement with Arp's (1960a) for the SMC of $-2.47(\mathrm{~V})$ and $-2.25(B)$ since, as we see in Fig. 8, a small change in slope can mean a large numerical change. Furthermore, Arp's work is based primarily on the shorter

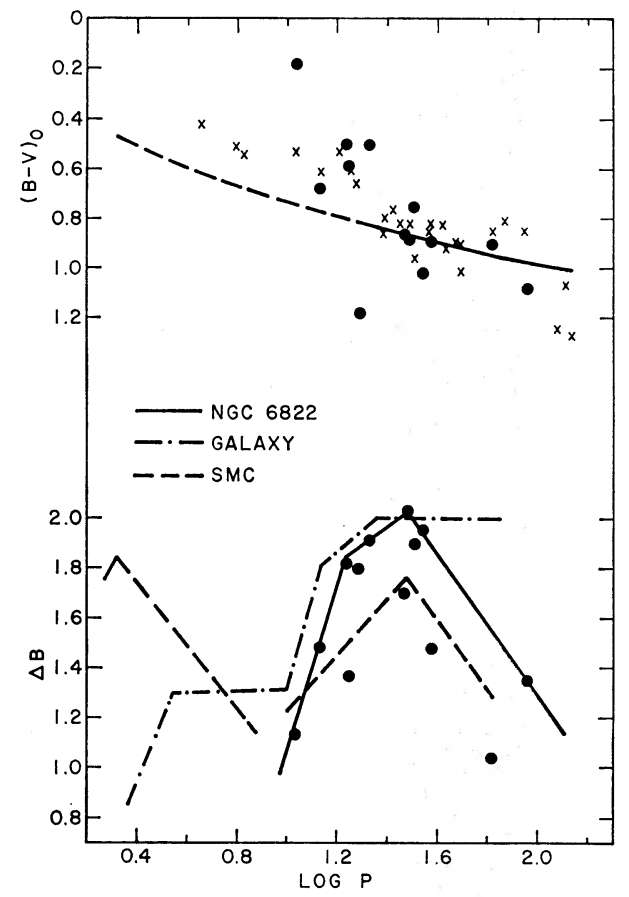

FIG. 7. The Cepheid period-color and period-amplitude relations. Circles are for variables from NGC 6822; crosses are those in the Magellanic Clouds. 


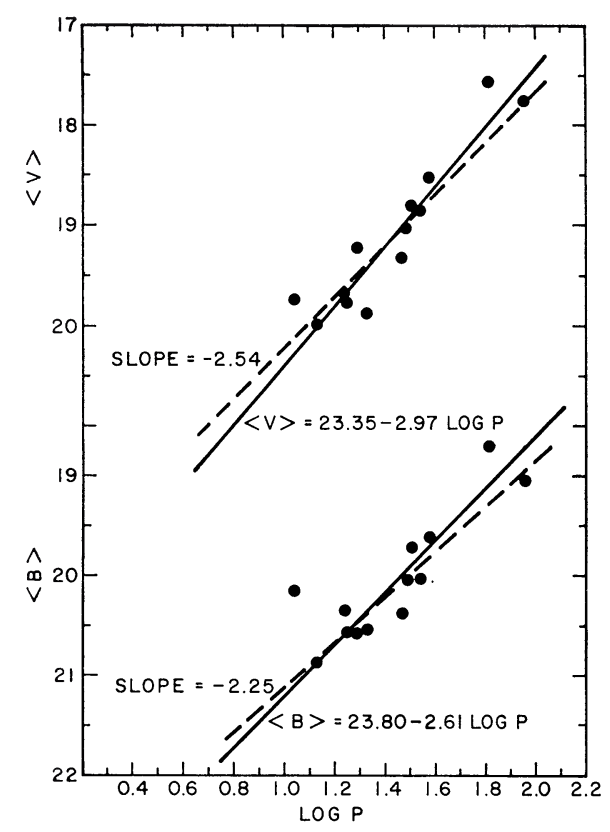

FIg. 8. The Cepheid period-luminosity relation. Full lines are relations found here. Dashed lines are Arp's SMC slopes.

period Cepheids. It is possible that the period-luminosity line curves toward a steeper slope at longer periods. The Gascoigne and Kron slopes, being based on a wider range of periods than here and twice as many stars, were fitted to the NGC 6822 points, producing the period-luminosity relations:

$$
\begin{aligned}
& \langle V\rangle=23.35-2.97 \log P, \\
& \langle B\rangle=23.80-2.61 \log P .
\end{aligned}
$$

To get the distance modulus, the five galactic cluster Cepheids (Kraft 1961b) were added to the $\langle V\rangle-\log P$ line. The best fit was obtained (by eye) with a distance modulus of $24.56 \pm 0.07$, assuming the line has no curvature. This corresponds to an unreddened true modulus of

$$
\begin{aligned}
(m-M)_{0} & =23.75 \pm 0.15, \\
r & =560 \mathrm{kpc} .
\end{aligned}
$$

With this zero point, the absolute period-luminosity relations are:

$$
\begin{aligned}
\left\langle M_{\mathrm{v}}{ }^{0}\right. & =-1.20-2.97 \log P, \\
\left\langle M_{B}{ }^{0}\right\rangle & =-1.03-2.61 \log P .
\end{aligned}
$$

This is consistent with a distance modulus for the Clouds of 19.15 , for $0^{\mathrm{m}} \cdot 05$ reddening.

\section{THEORY}

Because the stars of NGC 6822 were formed over at least $3 \times 10^{7} \mathrm{yr}$, we cannot apply theory here as we do to determine the age of clusters. Evolved stars of all initial luminosities are represented, so that we must know not only the initial luminosity function, but also the time-dependent rate of star formation in order to explain the present situation.

In Fig. 9, evolutionary paths have been superposed on a schematic color-magnitude diagram of NGC 6822. The short tracks for the very massive stars (121.1 and 62.7) have been taken from Schwarzschild and Härm (1958), the 3097 $\odot$ track is by Stothers $(1963,1964$, 1966), the long track (broken line) of a $15.697 \odot$ star was calculated by Hayashi and Cameron (1961, with additional stages reported by Hayashi and others, 1962), and the two at 15 and $997 \odot$ are by Iben (1966). The age-zero main sequence is indicated. All the calculations were for Population I stars with $(X=0.7$, $Y=0.3, Z=0.02)$ except for that at 15.6 which had a composition $(X=0.9, Y=0.1, Z=0.02)$. Hayashi remarks that use of the population I mixture would shift the red limit of the $15.697 \odot$ zigzag (beginning of helium burning) from $(B-V)_{0}=0^{\mathrm{m}} \cdot 20$, to $-0^{\mathrm{m}} \cdot 14$; with this change, we see the two tracks at 15 and 15.6 are very similar. The 15.6 path includes carbon burning, but the 30,15, and $991 \odot$ models were stopped at helium depletion. All the tracks were transformed from $\left[M_{\mathrm{bol}}-\log T_{e}\right]$ to the $\left[M_{\mathrm{v}}-(B-V)_{0}\right]$ plane by the relations from Arp (1958), amended to produce smaller bolometric corrections for cool luminosity class I stars (Arp, private communication).

No evolutionary path calculated has reached colors as red as observed here. Although this may be a spurious effect due to an incorrect $T_{e}-(B-V)$ transformation, it is more likely that either the models have not been continued long enough, or else some physical factor has not been included. From the number of stars involved, the red stage must last a considerable fraction of the post-main sequence lifetime. Neutrino losses may play an important part here. Iben has suggested that such losses might act as a refrigerant, cooling the

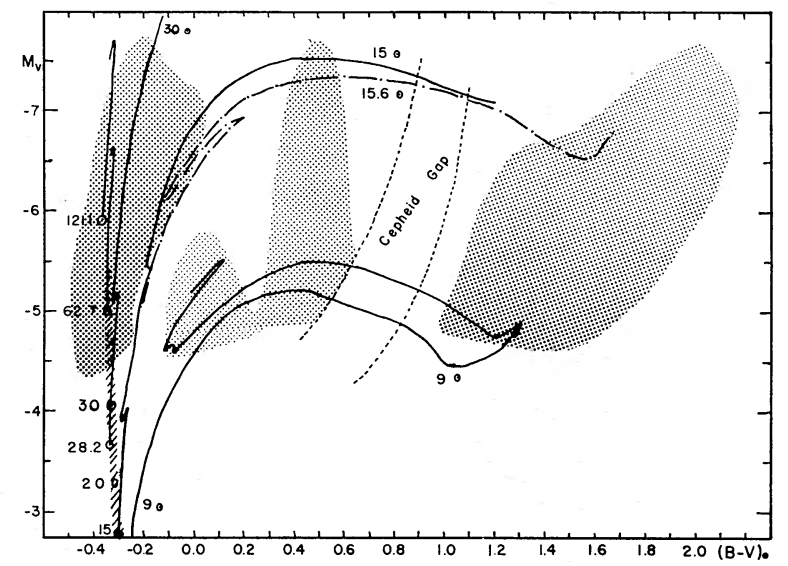

FIG. 9. Schematic color-magnitude diagram of NGC 6822 with evolutionary tracks. Hatching indicates the main sequence. 


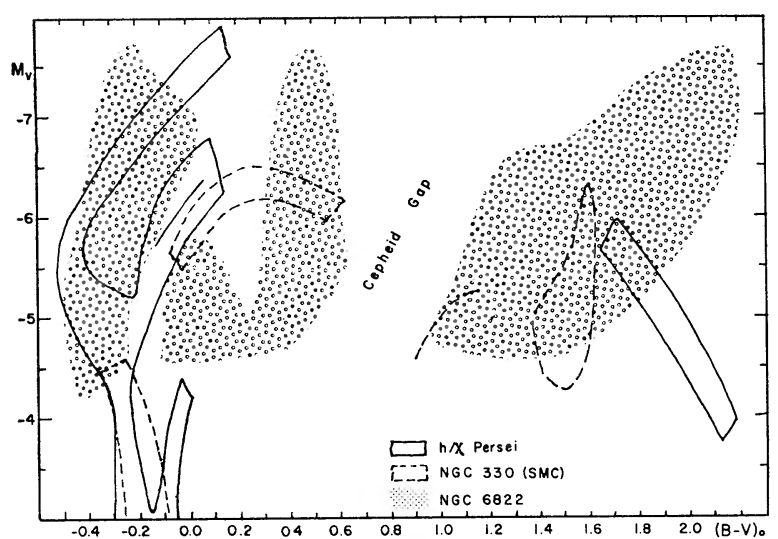

FIG. 10. Schematic color-magnitude diagrams of NGC 6822, h/ $\chi$ Persei, and NGC 330.

gravitationally contracting core during the $\mathrm{He}$ and $\mathrm{H}$ shell-burning phase, which may prolong the time spent on.' the red giant branch. Inclusion of this effect in models of massive stars may yield, through comparisons with systems like NGC 6822, empirical confirmation of the theory of neutrino interactions.

The use of the brightest stars in a galaxy as distance indicators depends on the uniformity of the luminosity function in all nebulae. As we have seen from the discussion of stellar content (illustrated in Fig. 10) the blue sequences are similar in all systems except insofar as a system, through age, has lost its very brightest stars. On a galactic scale, star formation will be continuous in time, at least to the extent of populating the main sequence up to $-8 \mathrm{mag}$ (in $M_{B}$ ). Sandage (1958), in his discussion of the distance scale, points out that the brightest stars in the Large Cloud, at $-9.8,-9.5,-10.1$, are one magnitude brighter than those in the Small Cloud $(-8.8,-8.5)$ and M31 (-8.3). In our own Galaxy we have No. 12 VI Cygni at -9.6 (Sharpless 1957). For NGC 6822, the three brightest stars are $\mathrm{C} 74, \mathrm{C} 84$, and $\mathrm{D} 156$, with $M_{B}$ $=-8.27,-8.58$, and -9.05 , respectively.

The red sequences, unlike the blue, are different for each system. This may be because of age differences, which result in clusters having their red giants come from a small mass range (the particular range depending on the cluster age), whereas NGC 6822 has a wide variety of masses, including those of stars which have already burned out in the clusters we know. Possibly the composition may vary slightly between systems. For small differences, the hydrogen-burning stage is relatively unaffected, but the surface temperature of red supergiants is very sensitive to the previous evolution because of mixing by its deeply convective envelope. Since $B-V$ is a steep function of $\log T_{e}$ at low temperatures, large variations in color may well result from a small chemical difference. This nonuniformity of the red supergiants will not create uncertainty in the use of bright stars as distance indicators for far-off galaxies because, on the blue-sensitive plates used to pick out the most luminous stars, the red sequence is two magnitudes fainter than the blue.

\section{CONCLUSION}

We have seen that NGC 6822 has a sufficiently close resemblance to other systems previously studied, that we may gain confidence in our knowledge of their properties as a group. Its brightest stars are of comparable luminosity, its Cepheid period-color-luminosity relation shows agreement, the color-magnitude diagram has similar outlines, with those in our own Galaxy, in the Magellanic Clouds, and in M31. We may, therefore, treat its differences as exceptions which prove the rules of general uniformity.

The most noticeable difference is the presence of the many bright red supergiants and the high percentage of variable stars found among them. Intensive observation of the few local red supergiants and models of far advanced evolutionary stages may explain them. The important problem of the space location of the hump stars may be further attacked by measuring a color-magnitude diagram in a field offset from the nebula by $1^{\circ}$, as well as by radial velocity measures of the analogous stars in the Clouds. If they are extrinsic to our Galaxy, present evolutionary theory has no interpretation for them.

It is reassuring to see that, to within the uncertainties of the data, there is no inconsistency in assuming a common Cepheid period-luminosity relation for the Clouds, NGC 6822, and our Galaxy. For M31, the published slope is still that previously found by Arp in the SMC. Although exactly identical relations cannot be expected, we may put reliance on distances obtained using a mean slope, to $\pm 15 \%$. The natural dispersion in the relation and its curvature (if there is any) are at present the causes of the greatest uncertainties in using it. Discovery of new Cepheids in the nebula will help define the intrinsic limits.

Further study of the fainter stars in NGC 6822 will be rewarding. Use of a large telescope in the southern hemisphere may be called for, since reducing the zenith angle will result in a more accurate magnitude scale and also in fainter plate limits. The glimpse we have now of the color-magnitude diagram is just large enough to be tantalizing. Addition of even one fainter magnitude may resolve some of the questions raised in this study.

\section{ACKNOWLEDGMENTS}

It is a pleasure to thank Dr. Halton C. Arp for his assistance and guidance, and the staff of the Mount Wilson and Palomar Observatories for the facilities they placed at my disposal.

This paper exists in complete form as Lawrence Radiation Laboratory report UCRL-14801-T, including 
a listing of the photographic plates used (Table 2), the colors and magnitudes $(V>19.5)$ of all nonvariable stars in the main body of the nebula (Table 3), finding charts for the stars in Table 3 (Plates II-VII), and a tabulation of the variable star magnitudes from each photographic plate (Table 10). Copies of the report are available from the author and from the laboratory.

\section{REFERENCES}

Allen, C. W. 1963, Astrophysical Quantities (Athlone Press, London), 2nd ed.

Arp, H. C. 1958, Handbuch der Physik (Springer-Verlag, Berlin), LI, p. 75.

- 1959, Astrophys. J. 129, 507.

. 1960a, Astron. J. 65, 404 .

1960b, Astrophys. $J$. 133, 869

1962, ibid. 135, 971

Bok, B. J. 1931, Harvard Circ. No. 371.

Bok, B. J., Bok, P. F., and Basinski, J. M. 1967, Monthly Notices Roy. Astron. Soc. (to be published).

Campbell, L. 1939, Pop. A stron. 47, 277.

de Vaucouleurs, G. 1964, Reference Catalogue of Bright Galaxies (University of Texas Press, Austin, Texas).

Fernie, J. D. 1963, Astron. J. 68, 780 .

Gascoigne, S. C. B., and Kron, G. E. 1965, Monthly Notices Roy. Astron. Soc. $130,23$.

Holmberg, E. 1957, Medd. Lund, Ser. II No. 136.

Hubble, E. 1925, Astrophys. J. 62, 409.

Hayashi, C., and Cameron, R. 1961, ibid. 136, 166.

Hayashi, C., Hōshi, R., and Sugimoto, D. 1962, Progr. Theoret. Phys. Suppl. No. 22.
Iben, I. 1966, A strophys. J. 143, 505 and 516.

Johnson, H. L. 1951, ibid. 114, 522.

- 1953, ibid. 117, 322.

. 1958, Lowell Bull. 90, IV, 37.

Johnson, H. L., and Iriarte, B. 1958, ibid. 91, IV, 47.

Kraft, R. 1961a, A strophys. J. 133, 39. 1961b, ibid. 134, 616.

Kukarkin, B. V., and Parenago, P. P. 1958, General Catalogue of Variable Stars ("P. K. Sternberg" State Astronomical Institute and "N. V. Lomosov" Moscow State University, Moscow), 2nd ed.

Limber, D. N. 1960, Astrophys. J. 131, 168.

Plaut, L., and Soudan, A. 1962, Bull. Astron. Inst. Neth. XVII, 70. Sandage, A. 1958, Astrophys. J. 127, 513.

Schwarzschild, M., and Härm, R. 1958, ibid. 128, 348.

Seares, F. H., van Rhijn, P. J., Joyner, M. C., and Richmond, M. L. 1925, Astrophys. J., 62, 320.

Sérsic, J. L. 1959, Observatory 79, 54.

Shapley, H. 1943, Galaxies (Blakiston Company, Philadelphia, Pennsylvania).

Sharpless, S. 1957, Publ. Astron. Soc. Pacific 69, 239.

Starikova, G. A. 1960, Soviet Astron.-AJ 4, 451.

Stebbins, J., Whitford, A. E., and Johnson, H. L. 1950, A strophys. J. 112, 469 .

Stothers, R. 1963, ibid. 138, 1074. 1964, ibid. 140, 510 1966, ibid. 143, 91.

Swope, H. 1963, Astron. J. 68, 435.

Wildey, R. L. 1963, ibid. 68, 190.

-. 1964, Astrophys. J. Suppl. 8, 439.

Wooley, R. v.d.R. 1963, Roy. Obs. Bull. No. 66.

van Rhijn, P. J. 1929, Groningen Publ. No. 43.

Volders, L., and Högbom, J. A. 1961, Bull. Astron. Inst. Neth. XV, 307. 


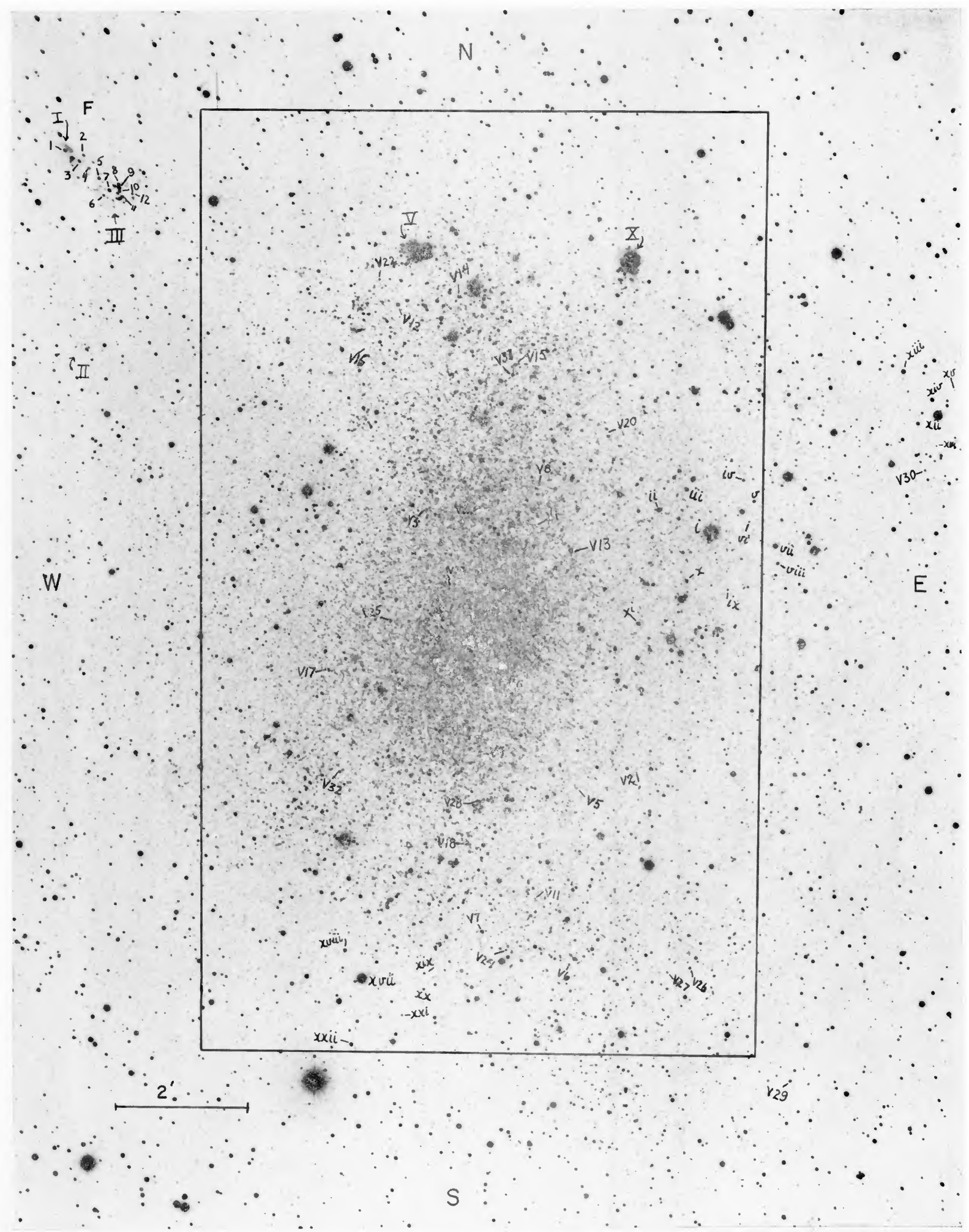

Plate II. (No. 1, Kayser) NGC 6822: Photoelectric standards and variables. Large Roman numerals indicate emission regions. (PH-433-B.) 